\title{
Flow-eontrolled ventilation - a new and promising method of ventilation presented with a review of the literature
}

\author{
Szymon Bialka', Piotr Palaczynski', Konstanty Szuldrzynski², Piotr Wichary ${ }^{3}$, Denis Kowalski³, \\ José W.A. van der Hoorn ${ }^{4}$, Lena Böttinger ${ }^{4}$, Hanna Misiolek'
}

${ }^{1}$ Department of Anaesthesiology, Intensive Care and Emergency Medicine, Faculty of Medical Sciences in Zabrze,

Medical University of Silesia, Katowice, Poland

${ }^{2}$ Department of Anaesthesiology and Intensive Care, Central Clinical Hospital Ministry of Interior and Administration,

Warsaw, Poland

${ }^{3}$ Student Scientific Society at the Department of Anaesthesiology, Intensive Care and Emergency Medicine, Faculty of Medical

Sciences in Zabrze, Medical University of Silesia, Katowice, Poland

${ }^{4}$ Ventinova Medical B.V., Eindhoven, The Netherlands

\begin{abstract}
Substantial efforts have been undertaken to identify and minimise factors responsible for the development of ventilator-induced lung injury. A novel approach to this problem addresses energy dissipated in lung tissue during the breathing cycle as one of the key problems. Flow-controlled ventilation is a new modality of mechanical ventilation based on a constant flow during both inspiration and expiration. This review aims to evaluate the current evidence available regarding flow-controlled ventilation. Lastly, three cases of flow-controlled ventilation application are presented: ventilation with a small lumen tube during tracheal resection, one-lung ventilation during thoracoscopic lobectomy, and ventilation of a critically ill patient with acute respiratory distress syndrome in an intensive care unit setting.
\end{abstract}

Key words: mechanical ventilation, ventilator-induced lung injury, flow-controlled ventilation.
Anaesthesiol Intensive Ther 2022; 54, 1: 62-70

Received: 12.07.2021, accepted: 16.11.2021

\section{CORRESPONDING AUTHOR:}

Szymon Bialka, Department of Anaesthesiology, Intensive Care and Emergency Medicine, Faculty of Medical Sciences in Zabrze, Medical University of Silesia, Katowice, Poland, e-mail: szymon.bialka@gmail.com

\section{INTRODUCTION}

\section{Ventilator-induced lung injury}

In recent years substantial efforts have been undertaken to identify and minimise factors responsible for ventilator-induced lung injury (VILI). Barotrauma related to high plateau pressures, volutrauma caused by high tidal volumes and atelectrauma associated with cyclic opening and closing of alveoli represent some of the most important triggers of VILI [1]. However, those factors do not encompass all the phenomena responsible for the damage of lung parenchyma.

\section{Energy dissipation}

A major paradigm shift was brought by the introduction of the driving pressure concept, which enabled improvement of treatment outcomes [2]. The latest reports indicate that the energy the ventilator delivers to the respiratory system may explain VILI more comprehensively [3]. Consequently, a decrease of dissipated mechanical energy in the lungs may translate into minimisation of lung damage. Mathematical models have proven that ventilation with constant and continuous flow during both in- spiration and expiration with an equal inspiration to expiration ratio adheres to these principles [4]. A passive expiration that occurs with a high and instable flow is therefore a source of energy dissipation.

\section{Ventilation through a small bore lumen}

A method of controlling expiratory flow was described for the first time in 2010 and was called expiratory ventilation assistance (EVA). This was a new ventilation method with a fully controlled ventilation cycle, consisting of a constant linear inward and outward gas flow [5]. Exhalation was made controllable by adding resistance in the respiratory circuit and creating expiration by suction (by means of the Venturi effect). Initially, this technique was developed to provide complete ventilation in critically obstructed airways through an extremely thin lumen (ID $2.0 \mathrm{~mm}$ ) using the manual ventilator Ventrain (Ventinova Medical BV, Eindhoven, The Netherlands; Figure 1). Ventrain has proven to be life-saving via both the subglottic route (needle cannula) and in combination with an orally placed thin tube (e.g. tube changer, bronchoscope) [6-10]. 

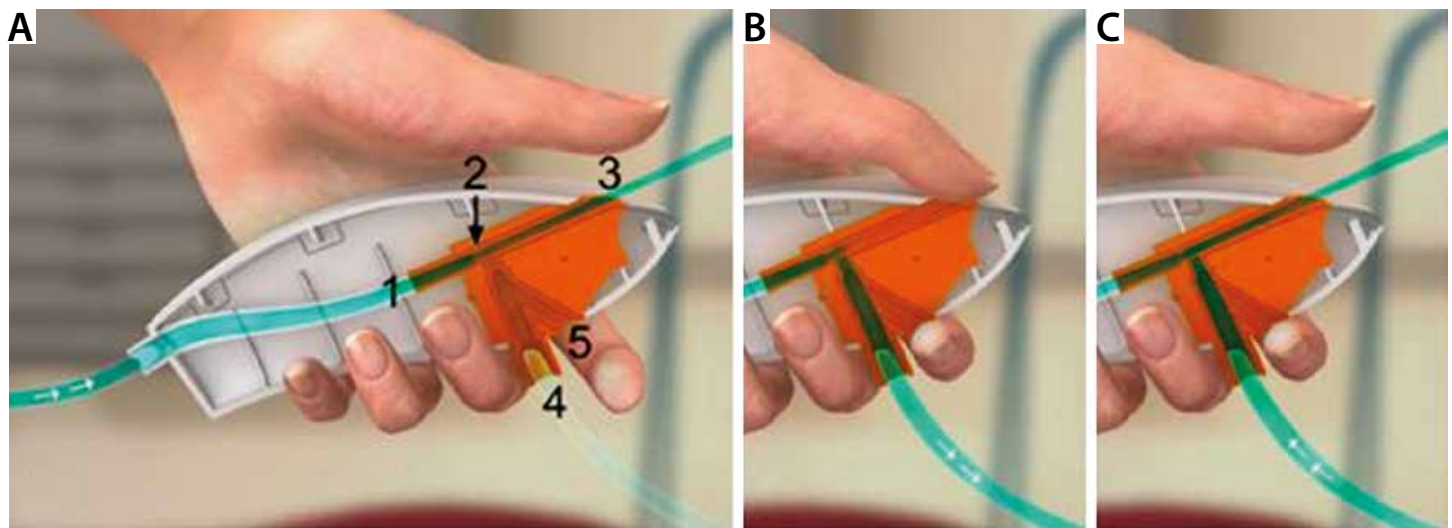

FIGURE 1. Working mechanism of Ventrain. A) Oxygen inlet from high pressure source with flow modulator (1), oxygen flow is accelerated by the narrowing nozzle (2) and exits Ventrain (3), creating a sub-atmospheric pressure at port (4). This port is connected to a small bore lumen (e.g. transtracheal catheter, Tritube) inside the patient's airway. A bypass (5) is designed to let air in and will activate the device when this bypass is closed. B) Closing port (3) creates an insufflation via (4). C) Opening (3) creates an exhalation (Copyright: Ventinova Medical B.V.)

In addition to the highly effective reoxygenation during hypoxia [11-13], this technique has added value in laryngological surgery [14-17] and one-lung ventilation [18] because of the possibility to ventilate patients through a thin tube or catheter [19]. Of note, efficient ventilation - thus, both oxygenation and $\mathrm{CO}_{2}$ removal - requires a closed airway.

\section{Linearised expiration flow}

Computer-controlled resistors decelerating and linearising expiratory flow assessed in animal models provided insights into the respiratory system mechanics [20]. The working principle of these systems is based on partial occlusion of the ventilator's expiratory outlet that decelerates initial high expiratory flow to moderate flow through the expiration phase. In a porcine model with artificially induced lung injury it was shown to improve lung function and mechanics with attenuated lung damage compared with standard volume-controlled ventilation (VCV) [21]. Furthermore, in healthy lungs expiration with decelerated and linearised flow has been shown to improve lung aeration [22]. Similar results were obtained in clinical trials, where lung healthy patients with a bandaged thorax simulating restrictive lung disease reported more comfortable breathing with restricted expiration flow compared to breathing through a mouthpiece connected to a ventilator with positive end-expiratory pressure (PEEP) [23]. Also, this module was proven to be a safe and viable method in patients undergoing neurosurgical procedures, improving ventilation in dorsal areas and thus leading to more homogeneous regional ventilation [23].

Furthermore, the potentially deleterious effect of passive expiration was shown by the group of Kavanagh. They demonstrated in a rat model that an abrupt lung deflation after sustained inflation could cause acute lung injury [24].

\section{Flow-controlled ventilation: fully controlling the expiration flow}

The possibility of being able to control the entire ventilation cycle and at the same time ventilate through a thin ventilation tube led to the logical need to apply this ventilation technique in an automated fashion. EVA was further developed into the mechanical ventilation method: flow-controlled ventilation (FCV), which is clinically available using an Evone ventilator (Ventinova Medical BV, Eindhoven, The Netherlands). This innovative method of ventilation was created by Dietmar Enk.

The FCV mode of the Evone ventilator is used for full ventilation while controlling the flow throughout the whole ventilation cycle. This is established by generating a continuous flow into the patient's lungs, followed by a continuous negative flow, sucking gasses out of the lungs during expiration. Unlike previous methods of applied resistance during expiration, in this case the expiration is an active process. To efficiently control expiration flow, FCV depends on a high-resistance breathing circuit, which is provided either by use of the ultrathin endotracheal cuffed tube Tritube (Ventinova Medical BV, Eindhoven, The Netherlands) or by connecting the conventional tube adapter to a conventional adult endotracheal tube. The FCV ventilation cycle relies on accurate intratracheal pressure measurements and is governed by four operator settings, as illustrated in Figure 2: 1) end-expiratory pressure (EEP), 2) peak inspiratory pressure, 3) inspiration flow, 4) inspiration to expiration ( $\mathrm{I}: \mathrm{E})$ ratio. Thus, in contrast to conventional ventilation modes, the inspiratory tidal volumes and respiratory rate cannot be directly set by the operator, but are dependent on pressure settings, flows and respiratory system compliance. Similar to other devices, $\mathrm{FiO}_{2}$ (fraction of inspired oxygen) can be set directly. Additionally, 


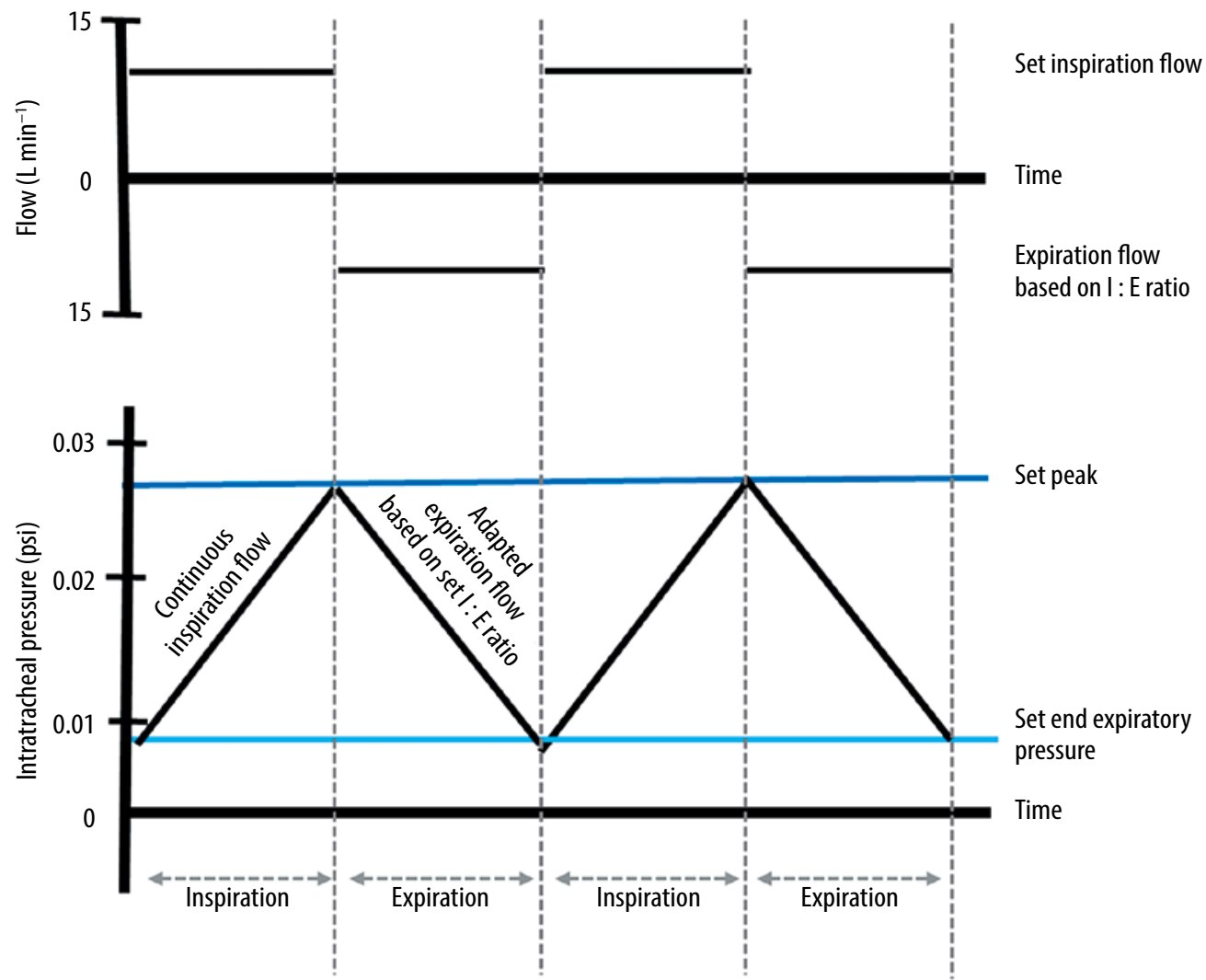

FIGURE 2. Setting flow-controlled ventilation requires four parameters: 1) end-expiratory pressure, 2) peak, 3) inspiration flow and 4) I: E ratio. At the set flow rate gas is insufflated from end-expiratory pressure (EEP) until it reaches peak level. Then the flow is reversed and sucked out at the rate to reach the set I: E ratio until EEP is reached. The next insufflation follows (Copyright: Ventinova Medical B.V.)

Evone provides a high-frequency jet ventilation mode, which is meant to be performed solely via a small lumen endotracheal tube with a deflated cuff to reduce the risk of barotrauma. This mode is used primarily in the postoperative period when the patient begins to breathe spontaneously and is being prepared for extubation. The physician sets the frequency, inspiration percentage, and driving pressure according to the needs of the patient.

FCV is based on a constant inspiratory and expiratory gas flow, whereby the desired ventilation pressures (PEEP and Peak) are preset by the user. The constant gas flows cause a linear rise and fall of intratracheal pressures (Figure 3). There are no significant periods of no flow, as are known during VCV and pressure-controlled ventilation (PCV) [25]. The expiratory pressure drop in the airway is thus significantly milder with FCV than with VCV and PCV.

The first multicentre observational study in which FCV was applied clinically during ENT surgery was published in 2019 [26]. FCV ventilation with Evone through the thin tube Tritube resulted in adequate et $\mathrm{CO}_{2}$ levels, achieved with relatively low minute volumes. The thin breathing tube turned out to be a valuable extension of the airway surgery toolbox [26].
As further explained below, FCV results in a more homogeneous and efficient ventilation and presents a lower risk of lung damage than the conventional ventilation forms VCV and PCV. In addition, FCV has major (surgical) advantages during laryngeal surgery.

\section{Flow-controlled ventilation: ventilation through a thin endotracheal tube}

FCV can be applied using a Tritube, a thin cuffed endotracheal tube (Figure 4). With an outer diameter of only $4.4 \mathrm{~mm}$, it offers advantages during earnose-throat (ENT) surgery. The first series of patient cases was published by Kristensen et al. [14]. During laryngeal surgery, the use of a Tritube in combination with Ventrain resulted in "unprecedented visibility of the intubated airways during oral, pharyngeal, laryngeal or tracheal procedures". In this series they also described two awake intubations in highly complex patients, which were later also done, somewhat modified, by others [27].

Two randomised controlled trials confirm the advantages of the Tritube over an MLT-6 (Microlaryngeal Tube 6.0; Shiley, Medtronic, Meerbusch, Germany) tube during laryngeal surgery.

In the first study in patients with a non-complex airway, the Tritube showed a greater proportion of 

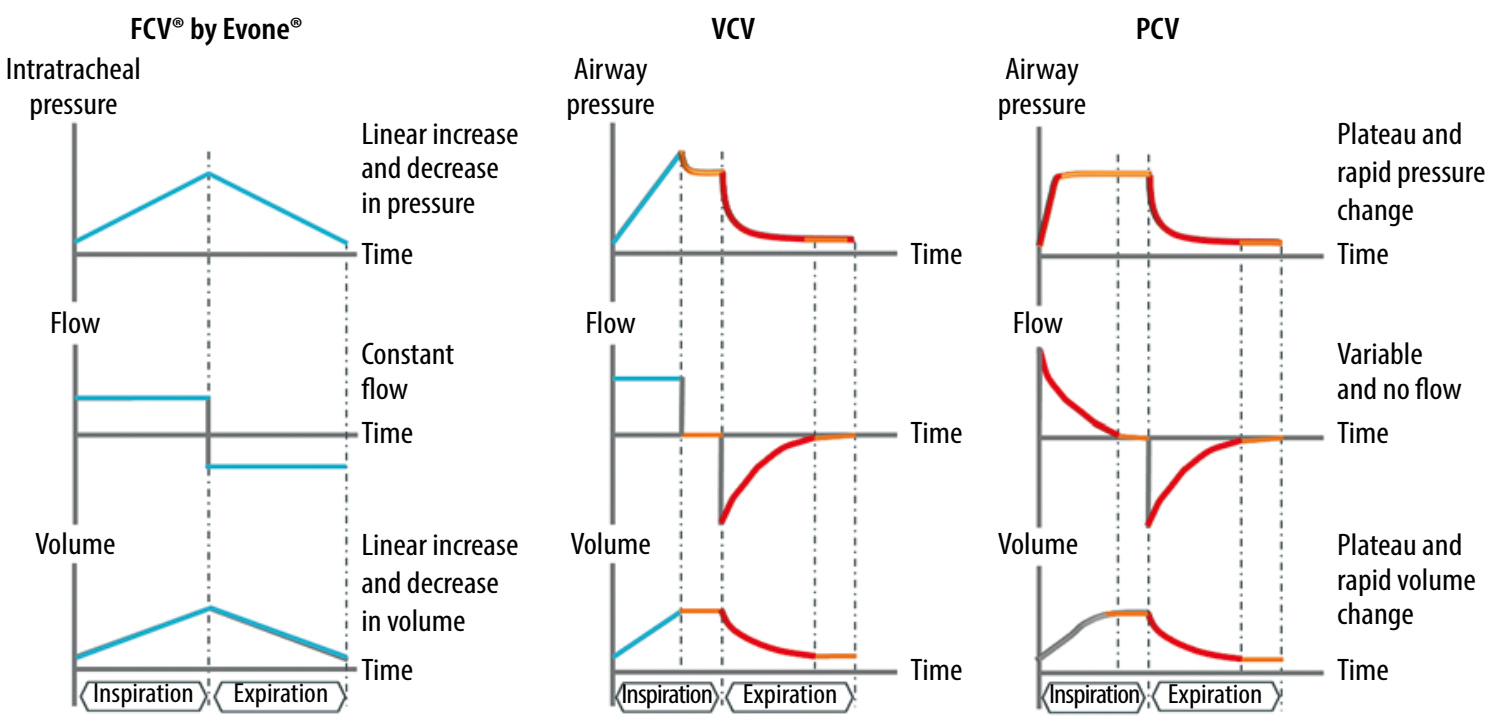

FIGURE 3. Volume, flow and pressure graphs during inspiration and expiration for flow-controlled ventilation (FCV), volume-controlled ventilation (VCV) and pressure-controlled ventilation (PCV) (Copyright:Ventinova Medical B.V.)

laryngeal structures visible to the surgeon $(93 \%$ vs. $78 \%, P<0.001)$, leading to improved surgical conditions especially for less experienced surgeons $(P=0.006)$ [28]. Kristensen et al. [29] included more complex patients with a predicted difficult airway. In these patients, intubation conditions, surgical conditions and visibility of the larynx were significantly improved $(P=0.004)$. In patients at high risk of postoperative airway problems (e.g. after complex laryngeal/tracheal surgery), it may be important to maintain the airway postoperatively until the risk of these problems has normalised. The Tritube also seems to have added value for these situations. Kristensen et al. [29] reported that postoperatively the Tritube with an empty cuff is at least as well tolerat-

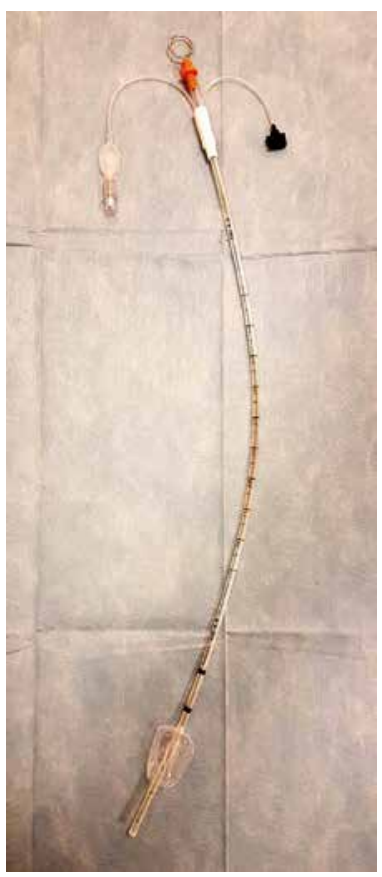

FIGURE 4. Tritube (Ventinova Medical BV, Eindhoven, Netherlands) is equipped with a mouldable stylet; on the left a cuff lumen used to inflate and deflate the balloon is presented. On the right an intratracheal pressure measurement lumen used for continuous intratracheal pressure measurements that will be connected to the Ventinova respirator is seen (Ventinova Medical BV, Eindhoven, Netherlands) ed as a tube changer. Patients were able to breathe and talk freely while still intubated with the Tritube.

A recognised "thin tube" ventilation method is jet ventilation, which is based on the application of high pressures and thus requires an open airway to prevent barotrauma. As a result, jet ventilation has recognised drawbacks such as moving anatomy due to backflow, poor $\mathrm{CO}_{2}$ elimination, an aspiration risk and contamination risk for the surgeon. The exact advantages of FCV over jet ventilation have not been evaluated in a study, but seem evident as FCV uses a cuffed tube that protects against aspiration and prevents moving anatomy, and with its use $\mathrm{CO}_{2}$ elimination is proven to be effective.

This reasoning was recently confirmed by Meulemans et al. [30], who used the Tritube and Evone in 15 patients undergoing upper airway surgery.

\section{Flow-controlled ventilation: a more efficient ventilation}

The effective $\mathrm{CO}_{2}$ elimination, increased ventilation efficiency and improved lung recruitment of FCV over VCV have been demonstrated in several randomised clinical trials. During laryngeal surgery, FCV led to better lung aeration and lung compliance $\left(61 \pm 10 \mathrm{~mL} \mathrm{cmH}_{2} \mathrm{O}^{-1}\right.$ in FCV group vs. 46 $\pm 5 \mathrm{~mL} \mathrm{cmH}_{2} \mathrm{O}^{-1}$ in VCV group, $P<0.001$ ) [28]. Also in (morbidly) obese patients FCV showed improved lung recruitment compared to VCV $(P<0.001)$ [31]. In two studies in lung healthy patients, FCV resulted in more efficient ventilation with better $\mathrm{CO}_{2}$ removal and one also showed better oxygenation (+9\%, $P<0.001)[32,33]$.

These results are in line with previously performed preclinical studies. In lung healthy pigs, FCV improved arterial oxygenation ( $+9 \%, P=0.002)$, requiring a lower minute volume $(-21 \%, P=0.02)$ 
for normoventilation [34]. Computed tomography (CT) analysis showed this to be related to improved lung recruitment. The results were even more striking when FCV was applied in an acute respiratory distress syndrome (ARDS) pig model (46\% improved oxygenation, $P=0.035 ; 26 \%$ lower minute volume, $P<0.001$ ) [35], or when FCV was individually optimised and compared to PCV (24\% improved oxygenation, $P=0.0097,53 \%$ lower minute volume, $P<0.001$ ) [36]. Also in these animals FCV prevented atelectasis and improved ventilation, especially in dorsal lung parts. In addition, significantly less lung damage was measured in the FCV ventilated animals in the lung disease: the alveolar wall thickness was smaller (7.8 $\pm 0.2 \mathrm{~m}$ vs. $5.5 \pm 0.1 \mathrm{~m} ; P<0.001)$ and there was less cell infiltrate ( $32 \pm 2$ vs. $20 \pm 2 /$ field; $P<0.001$ ) [35].

\section{Flow-controlled ventilation - lung damage limitation}

As shown in animal studies, linearisation of expiratory flow has lung protective effects [21, 35]. The constant and continuous flow during FCV results in less shear stress compared to VCV and PCV, thereby greatly reducing the amount of energy dissipation. The amount of energy generated during FCV per inhalation and exhalation is just sufficient for ventilation of the patient. In other words, little energy is absorbed in the lungs, which could cause damage to the lung tissue. Ventilation is thus possible with a significantly reduced risk of lung damage.

Barnes et al. published theoretical physical evidence that to minimise energy dissipation gas flows should be constant and continuous with an I: E ratio close to one. They calculated that FCV ventilation leads to less energy dissipation than PCV $(-52 \%)$ and VCV (-32\%) [4]. The area of the pressure-volume loop (PV loop) represents the amount of energy dissipated. Unlike conventional ventilators, which calculate intratracheal pressures based on pressures measured on the ventilator side, Evone actually measures pressures intratracheally. This makes it possible to measure energy dissipation (area of PV loop) reliably, as done by Barnes and Enk [37] in a patient. The measured energy dissipation turned out to be remarkably low $\left(0.17 \mathrm{~J} \mathrm{~L}^{-1}\right)$ and even equal to values reported for spontaneous respiration $\left(0.2-0.7 \mathrm{~J} \mathrm{~L}^{-1}\right)$.

\section{CASE SERIES REPORT}

In all of the presented cases patients' consent to publication was obtained.

\section{Tracheal resection}

The first case of the presented series describes a 52-year-old woman admitted to the Thoracic Sur- gery Ward due to tracheal stenosis after a severe course of SARS-CoV-2 infection. Past medical history consisted of arterial hypertension and hypothyroidism. The patient was hospitalised in the COVID-19 Infectious Disease Ward in November 2020 with symptoms of viral pneumonia, and subsequently developed acute respiratory failure. After intubation mechanical ventilation was initiated and the patient was referred to the Intensive Care Unit (ICU). Mechanical ventilation was conducted for eight days. Following extubation and rehabilitation the patient was moved to the Internal Medicine Ward and then discharged home. Over the next weeks, the patient developed dyspnoea and tracheal stenosis was diagnosed with imaging studies and rigid bronchoscopy. The patient was qualified for tracheal resection and the small lumen endotracheal tube, flow-controlled ventilation with the Evone ventilator, and total intravenous anaesthesia (TIVA) were used. During the induction of anaesthesia direct laryngoscopy was performed and the airways were secured with a Tritube with no adverse events; the cuff of the endotracheal tube was placed beneath the stenosis. After initial stabilization of the respiratory system FCV parameters were titrated at PEEP $5 \mathrm{cmH}_{2} \mathrm{O}$ and $\mathrm{P}_{\text {peak }} 14 \mathrm{cmH}_{2} \mathrm{O}$ (peak pressure); the flow rate was set to $8 \mathrm{~L} \mathrm{~min}^{-1}$ with an I: E ratio of $1: 1, \mathrm{FiO}_{2} 0.4$. These settings enabled stable oxygenation and normocapnia throughout the procedure. No complications occurred during the intraoperative period. After the surgery the patient was extubated and a laryngeal mask (Aura Reusable Laryngeal Mask, Ambu, Denmark) was inserted as a means of securing the airways up to the point of full emergence. The attending surgeon described visibility and accessibility to the operative field as excellent; also no need for additional procedures such as crossfield intubation was seen as an advantage.

\section{Acute respiratory distress syndrome}

A 48-year-old male patient was admitted to the Intensive Care Unit after cervicotomy and bilateral thoracotomy due to mediastinitis and bilateral pleural empyema in the course of tonsillitis and perforation of an abscess to the retropharyngeal space. Rapid cardiopulmonary deterioration was observed before the admittance and immediate surgical intervention was needed. Past medical history consisted only of obesity. In the Intensive Care Unit, severe multisystem organ failure was observed despite prompt, multidimensional treatment. Due to the symptoms of ARDS including a low level of partial oxygen pressure $\left(\mathrm{PaO}_{2}\right)$ to fraction of inspired oxygen $\left(\mathrm{FiO}_{2}\right)$ ratio the use of flow-controlled ventilation and tube-in-tube (endotracheal tube with cuff, Ningbo Hi-Tech Unicmed, China) tech- 
nique was proposed for the next 24 hours. Initial mechanical ventilation was performed with the conventional pressure-controlled mode with PEEP $12 \mathrm{cmH}_{2} \mathrm{O}, \mathrm{P}_{\text {peak }} 26 \mathrm{cmH}_{2} \mathrm{O}, \mathrm{I}: \mathrm{E}(1: 1.8), \mathrm{f} 20 /$ per minute (number of breaths) with the Carescape R860 Ventilator (GE Healthcare, Chicago, USA); measured compliance was $16 \mathrm{~mL} \mathrm{cmH}_{2} \mathrm{O}^{-1}$. After the launch of $F C V$, introductory parameters were set similarly to PCV (PEEP $12 \mathrm{cmH}_{2} 0, P_{\text {peak }} 26, \mathrm{I}: \mathrm{E} 1: 1$ and flow of $\left.10 \mathrm{~L} \mathrm{~min}^{-1}\right)$; afterwards the $P_{\text {peak }}$ and PEEP were titrated according to lung mechanics measurements and capnography (Carescape, GE Healthcare, Chicago, USA), PEEP remained at $12 \mathrm{cmH}_{2} 0, \mathrm{P}_{\text {peak }}$ was reduced to $23 \mathrm{~L} \mathrm{~min}^{-1}$ and flow was modified to $14 \mathrm{~L} \mathrm{~min}^{-1}$. Sedation had to be significantly increased and a neuromuscular blocking agent was introduced to ensure proper ventilation. Within the designated time of ventilation slight improvement of compliance (22 $\mathrm{mL} \mathrm{cm} \mathrm{H}_{2} \mathrm{O}^{-1}$ ) along with a decrease of driving pressure was noted. Substantially reduced minute ventilation (from $16 \mathrm{~L} \mathrm{~min}^{-1}$ to $8.5 \mathrm{~L} \mathrm{~min}^{-1}$ ) and number of breaths procured a steady and gentle ventilation. Arterial blood gases and haemodynamic measurements using a Vigileo monitor (Edward Lifesciences, USA) were analysed and an increase in $\mathrm{pO}_{2}$ and constant level of $\mathrm{pCO}_{2}$ were observed; haemodynamic measurement parameters remained stable.

\section{Lobectomy with one-lung ventilation}

A 65-year-old woman underwent an elective video thoracoscopic lobectomy of the upper right lobe due to the malignant disease. Past medical history consisted of arterial hypertension, ischaemic heart disease, nephrolithiasis and nicotine addiction. Before the induction of general anaesthesia erector spinae plane block was performed with ropivacaine (Ropimol, Molteni, Italy) and lidocaine (Lidocaine, Grindeks, Latvia), induction of general anaesthesia was obtained with propofol (Propofol, Fresenius, Germany), fentanyl (Fentanyl WZF, Polfa, Poland) and cis-atracurium (Nimbex, Aspen Pharma, South Africa), a Robertshaw double lumen endotracheal tube size 39G (Double lumen tube, Teleflex, United Kingdom) was used to secure the airways, anaesthesia was maintained with a constant infusion of propofol and boluses of fentanyl and cisatracurium titrated to obtain adequate anaesthesia. Proper placement of the tube was confirmed with flexible bronchofibroscopy; initially conventional volume-controlled ventilation (VCV) was used, then when the need of left lung ventilation emerged, the Evone CTA (Conventional Tube Adapter, Ventinova Medical BV, Eindhoven, Netherlands) was connected to the left, bronchial lumen of the tube to allow initiation of flow-controlled ventilation. The initial optimally titrated parameters were set as follows: PEEP
$5 \mathrm{cmH}_{2} \mathrm{O}, \mathrm{P}_{\text {peak }} 16 \mathrm{cmH}_{2} \mathrm{O}, \mathrm{I}: \mathrm{E} 1: 1$, inspiratory flow of $10 \mathrm{~L} \mathrm{~min}^{-1}, \mathrm{FiO}_{2} 0.6$; later, due to sufficient oxygenation $\mathrm{FiO}_{2}$ was reduced to 0.4 , but an increase of $\mathrm{CO}_{2}$ level caused the need to elevate inspiratory flow to $12 \mathrm{~L} \mathrm{~min}^{-1}$ and $\mathrm{P}_{\text {peak }} 18 \mathrm{cmH}_{2} \mathrm{O}$. No complications were noted. After surgery, the patient was reintubated with a single lumen tube and was moved to the recovery room.

\section{DISCUSSION}

The above studies confirm the higher ventilation efficiency of FCV, the better lung recruitment, the potential to limit lung damage and the benefits of a thin cuffed tube during laryngeal surgery.

The scientific evidence for the clinical benefits for the patient is unequivocal but still limited due to the relatively small number of patients included in clinical studies. Larger, randomised controlled trials are needed to better demonstrate the (potential) benefits of FCV, as well as whether there are more limitations than described below.

The current COVID pandemic shines a new light on the strategies to manage (compromised) airways [38]. Also the incidence of laryngotracheal stenosis is expected to increase in relation to the frequent long term intubation of patients [39]. The value of the Tritube during tracheal resection is being evaluated (Trial NL7970 in Dutch clinical trial register). The Tritube would allow the surgeons to perform tracheal resections while it is left in place and without the need for cross field intubations, which lead to patient risk and aerosol generation. Also during tracheotomy procedures in the ICU aerosol reduction is key. A recent report described the performance of a tracheotomy procedure using a tube-in-tube technique (Tritube through a large-bore endotracheal tube), enabling minimal production of airway aerosols and thus increasing the safety of personnel, in addition to providing easy surgical access [40]. Furthermore, in cases of severe stenosis, the Tritube might prevent the need for ECMO during surgery, as described in a case of critical tracheal stenosis of $4 \mathrm{~mm}$ [41].

Relating to its ventilation effects, it is likely that FCV prevents atelectasis not only in morbidly obese patients, as has been shown [31], but also in other patients with an increased risk of compression atelectasis (e.g. Trendelenburg position, laparoscopy). In these patients, FCV may lead to fewer episodes of hypercapnia or desaturation and less need for high ventilation pressures. Patients with reduced ventilation capacity (e.g. during one lung ventilation) may also be better ventilated and oxygenated during surgery, which has already been shown in pigs [42]. This is currently being investigated in patients undergoing one lung ventilation (NCT04534933 in clinicaltrial.gov and DRKS00022198 in German 
Clinical trial register) Also, the improved lung recruitment by FCV may lead to benefits in patients undergoing cardiac surgery involving a cardiopulmonary bypass. When switching back to ventilation, the lungs must be recruited. Effects of FCV are being investigated in two clinical trials (NCT04306757 in clinicaltrials.gov and DRKS00018956 in the German clinical trial register). These currently ongoing studies primarily focus on oxygenation during surgery and inflammation parameters. However, FCV may also have implications for the patient's postoperative recovery, as intraoperative periods of desaturation, probably related to atelectasis, represent an important risk for the development of postoperative pulmonary complications [43].

Mechanical ventilation is a life-saving technology in acute respiratory failure. However, its use may lead to secondary damage of the lungs, specifically VILI. Several factors responsible for VILI have been described including mechanical energy dissipation within the lungs, atelectrauma and barotrauma. According to the existing evidence from animal and human studies, flow-controlled ventilation compared to conventional modes may provide at least equally efficient gas exchange and more homogeneous aeration of the lungs with decreased energy dissipation, and minimised risk of atelectrauma and barotrauma. This may make this technology a viable therapeutic option for patients developing ARDS during severe lung diseases such as SARS-CoV-2. Growing demand for the new means of treatment of patients in a critical condition led to the launch of clinical trials (NCT04399317 and NCT04894214 as registered in clinicaltrials.gov). However, still, large randomised clinical trials are warranted to assess the clinical usefulness and demonstrate the improved outcomes of patients in the intensive care unit setting.

One of the non-standard applications of the device is the possibility to set the negative end-expiratory pressure (NEEP) with the maximum value of -10 mbar $(-1 \mathrm{kPa})$. Theoretically, the effect of NEEP can be used during life-threatening hypovolaemic shock to increase cardiac output by reducing intrathoracic pressure and increasing the pressure gradient, allowing an improved venous return to the heart. This theory has been confirmed by preclinical data; however, its clinical effect is yet to be seen $[44,45]$.

Furthermore, FCV has been used for preservation of donor lungs during ex vivo lung perfusion (EVLP). FCV led to improved oxygenation and alveolar recruitment compared with VCV, with a higher proportion of well-aerated and less atelectatic lung areas [46]. Therefore, FCV may be a potential strategy to prolong EVLP over time.

Recently, it was hypothesised that minimization of the expiratory flow rate may be beneficial for both invasive and non-invasive mechanical ventilation of chronic obstructive pulmonary disease patients. Some of the symptoms' severity is attributed to central airway collapse during exhalation, which can be limited with application of counter pressure. However, clinical application of these effects is yet to be seen [47].

\section{LIMITATIONS}

One of the shortcomings of a small lumen tube is the risk of obstruction with excessive airway secretions or blood from the operating field. Patients with a difficult airway, in whom the intubation procedure may be traumatic and extend beyond the standard period, are especially susceptible to this risk. Meulemans et al. [30] recommend preoperative administration of 0.2 milligrams of glycopyrronium to reduce the secretion of mucus and prevent ventilation disturbance. This long-acting anticholinergic drug should be used with caution in the presence of diseases such as cardiac disorders, renal dysfunction, glaucoma or urinary retention. When faced with excessive secretions in the airway it is not possible to use a traditional suction catheter; however, with a deflated cuff the ventilation lumen can be used for suctioning, equivalent to a $12 \mathrm{Ch}$ catheter [15].

Another disadvantage arising from the cuffed small lumen tube is the risk of displacement from the trachea due to various reasons such as coughing, which might happen especially during emergence from anaesthesia. This situation may result in the need to switch to alternative methods of securing the airways and use of conventional ventilation modes which were reported in previous studies [14].

Furthermore, the extubation procedure itself in some cases may be challenging; thus Schmidt et al. proposed establishing protocols the attending anaesthetist may follow. In some cases FCV may be well tolerated up to the point of extubation; additional oxygen supplementation may be provided with a face mask. Another option is to deflate the cuff of the tube and switch to jet ventilation with an unsealed airway allowing spontaneous breathing. Another method is based on the use of an endotracheal tube as a subglottic oxygen insufflation catheter until the emergence ceases. Lastly, at the end of the intervention the patient may be switched to a standard airway device such as a laryngeal mask [14]. The ability to quickly change ventilation modes (with a sealed or unsealed airway) without changing the airway device provides a greater range of response options and additional ways to monitor the patient's status during unexpected events $[14,28]$.

However, it is not possible to use volatile anaesthetics during flow-controlled ventilation due to their physical characteristics. The need of total in- 
travenous anaesthesia and the current price of the small lumen tube, breathing circuit and essential accessories to conduct the ventilation procedure result in a much higher cost compared with a conventional approach [29]. As mentioned, the application of FCV to ventilate a patient is based on a clinical workflow that to some extent deviates from conventional ventilation strategies. Thus, sufficient training of users in both operation of Evone and clinical application of the FCV concept is required to ensure adequate ventilation and to enable optimal treatment of patients.

\section{CONCLUSIONS}

The concept of FCV is simple and at the same time innovative: by controlling both the inspiration phase and the expiration phase, the gradual natural respiration is simulated as closely as possible.

Flow-controlled ventilation provided by the Evone ventilator (Ventinova Medical BV, Eindhoven, The Netherlands), with the armamentarium of a specially designed small lumen endotracheal tube or, alternatively, an adapter for connection to conventional endotracheal tubes, seems to be a promising new multipurpose treatment option. Up-to-date clinical evidence focusses mainly on its use in short term ventilation in the operating theatre setting, proving its safety and feasibility and indicating ventilatory benefits. Moreover, FCV is unique in allowing the precise measurements of intratracheal pressures to assess the respective lung mechanics of a patient and provide individually optimised ventilation settings. The existing evidence and rationales and the ongoing clinical trials may provide evidence for its usefulness in the lung protective ventilation of patients with respiratory failure.

\section{ACKNOWLEDGEMENTS}

1. Financial support and sponsorship: none.

2. Conflicts of interest: José W.A. van der Hoorn and Lena Böttinger are employed by Ventinova Medical B.V., producer of the presented Ventrain, Tritube and Evone ventilator.

3. Presentation: part of the presented data was submitted as a poster presentation at EUROANAESTHESIA 2020 (Virtual Congress, 28-30 November 2020).

\section{REFERENCES}

1. Beitler JR, Malhotra A, Thompson BT. Ventilator-induced lung injury. Clin Chest Med 2016; 37: 633-646. doi: 10.1016/j.ccm.2016.07.004.

2. Bellani G, Laffey JG, Pham T, et al. Epidemiology, patterns of care, and mortality for patients with acute respiratory distress syndrome in intensive care units in 50 countries. JAMA 2016; 315: 788-800. doi: 10.1001/jama.2016.0291

3. Gattinoni L, Tonetti T, Cressoni M, et al. Ventilator-related causes of lung injury: the mechanical power. Intensive Care Med 2016; 42 1567-1575. doi: 10.1007/s00134-016-4505-2.
4. Barnes T, van Asseldonk D, Enk D. Minimisation of dissipated energy in the airways during mechanical ventilation by using constant inspiratory and expiratory flows - flow-controlled ventilation (FCV). Med Hypotheses 2018; 121: 167-176. doi: 10.1016/j.mehy.2018.09.038.

5. Hamaekers AEW, Götz T, Borg PAJ, Enk D. Achieving an adequate minute volume through a $2 \mathrm{~mm}$ transtracheal catheter in simulated upper airway obstruction using a modified industrial ejector. $\mathrm{Br} J$ Anaesth 2010; 104: 382-386. doi: 10.1093/bja/aep391.

6. Morrison S, Aerts S, Van Rompaey D, Vanderveken O. Failed awake intubation for critical airway obstruction rescued with the ventrain device and an arndt exchange catheter: a case report. A A Pract 2019: 13: 23-26. doi: 10.1213/XAA.0000000000000975.

7. Heuveling DA, Mahieu, HF, Jongsma-van Netten HG, Gerling V. Transtracheal use of the CriCath cannula in combination with the ventrain device for prevention of hypoxic arrest due to severe upper airway obstruction: a case report. A A Pract 2018; 11: 344-347. doi: 10.1213/XAA.0000000000001084

8. Escribá Alepuz FJ, Alonso García J, Cuchillo Sastriques JV, Alcalá E, Argente Navarro P. Emergency ventilation of infant subglottic stenosis through small-gauge lumen using the ventrain: a case report. A A Pract 2018; 10: 136-138. doi: 10.1213/XAA.000000000000657.

9. Wahlen, BM, Al-Thani H, El-Menyar A. Ventrain: from theory to practice. Bridging until re-tracheostomy. BMJ Case Rep 2017; 2017: bcr2017220403. doi: 10.1136/bcr-2017-220403.

10. Willemsen MGA, Noppens R, Mulder ALM, Enk D. Ventilation with the Ventrain through a small lumen catheter in the failed paediatric airway: two case reports. Br J Anaesth 2014; 112: 946-947. doi: 10.1093/bja/aeu125.

11. de Wolf MWP, Gottschall R, Preussler NP, Paxian M, Enk D. Emergency ventilation with the Ventrain ${ }^{\circ}$ through an airway exchange catheter in a porcine model of complete upper air-way obstruction. Can J Anaesth 2017; 64: 37-44. doi: 10.1007/s12630-016-0760-5.

12. Paxian M, Preussler NP, Rein T, Schlueter A, Gottschall R. Transtracheal ventilation with a novel ejector-based device (Ventrain) in open, partly obstructed, or totally closed upper air-ways in pigs. Br J Anaesth 2015; 115: 308-316. doi: 10.1093/bja/aev200.

13. Hamaekers AE, van der Beek T, Theunissen M, Enk D. Rescue ventilation through a small-bore transtracheal cannula in severe hypoxic pigs using expiratory ventilation assistance. Anesth Analg 2015; 120 : 890-894. doi: 10.1213/ANE.0000000000000584.

14. Kristensen MS, de Wolf MWP, Rasmussen LS. Ventilation via the $2.4 \mathrm{~mm}$ internal diameter Tritube ${ }^{\infty}$ with cuff - new possibilities in airway management. Acta Anaesthesiol Scand 2017; 61: 580-589. doi: 10.1111/ aas. 12894

15. Zuercher M, Pythoud-Brügger M, Kishore S, Schoettker P. Combined use of Ventrain and S-Guide for airway management of severe subglottic stenosis. Turk J Anaesthesiol Reanim 2019; 47: 238-241. doi: 10.5152/TJAR.2019.75428

16. Onwochei D, El-Boghdadly K, Ahmad I. Two-stage technique used to manage severe up-per airway obstruction and avoid surgical tracheostomy. A A Pract 2018; 10: 118-120. doi: 10.1213/XAA.000000 0000000645 .

17. Fearnley RA, Badiger S, Oakley RJ, Ahmad I. Elective use of the Ventrain for upper airway obstruction during high-frequency jet ventilation. J Clin Anesth 2016; 33: 233-235. doi: 10.1016/j.jclinane. 2016.03.024

18. Evers VM, Immink RV, van Boven WJP, van Berge Henegouwen MI, Hollmann MW, Veelo DP. Intraoperative use of the Ventrain for single lung ventilation after iatrogenic trauma to the left main bronchus during thoracoscopy: a case report. A A Case Rep 2017; 9: 116-118. doi: 10.1213/XAA.0000000000000542.

19. Doyle DJ. Ventilation via narrow-bore catheters: clinical and technical perspectives on the ventrain ventilation system. The Open Anesthesia Journal 2018; 12: 49-60. doi: 10.2174/2589645801812010049.

20. Schumann S, Goebel U, Haberstroh J, et al. Determination of respiratory system mechanics during inspiration and expiration by FLowcontrolled EXpiration (FLEX): a pilot study in anesthetized pigs. Minerva Anestesiol 2014; 80: 19-28.

21. Goebel U, Haberstroh J, Foerster K, et al. Flow-controlled expiration: a novel ventilation mode to attenuate experimental porcine lung injury. Br J Anaesth 2014; 113: 474-483. doi: 10.1093/bja/aeu058.

22. Borgmann S, Schmidt J, Goebel U, et al. Dorsal recruitment with flowcontrolled expiration (FLEX): an experimental study in mechanically ventilated lung-healthy and lung-injured pigs. Crit Care 2018; 22 : 245. doi: 10.1186/s13054-018-2168-9.

23. Wirth S, Springer S, Spaeth J, et al. Application of the Novel Ventilation Mode FLow-Controlled EXpiration (FLEX): a crossover proof- 
of-principle study in lung-healthy patients. Anesth Analg 2018; 125 : 1246-1252. doi: 10.1213/ANE.0000000000001991.

24. Katira BH, Engelberts D, Otulakowski G, et al. Abrupt deflation after sustained inflation causes lung injury. Am J Respir Crit Care Med 2018; 198: 1165-1176. doi: 10.1164/rccm.201801-0178OC.

25. Enk D. Method and Device for Ventilating a Patient. Available at: https://patentscope.wipo.int/search/en/detail.jsf?docId= WO2017148639.

26. Schmidt J, Günther F, Weber J, et al. Flow-controlled ventilation during ear, nose and throat surgery: a prospective observational study. Eur J Anaesthesiol 2019; 36: 327-334. doi: 10.1097/EJA. 0000000000000967

27. Jeyarajah K, Ahmad I. Awake tracheal placement of the Tritube under flexible bronchoscopic guidance. Case studies. Anaesthesia Cases 2018; 6: 1-5. doi: 10.21466/ac.ATPOTTU.2018.

28. Schmidt J, Günther F, Weber J, et al. Glottic visibility for laryngeal surgery: tritube vs. microlaryngeal tube: a randomised controlled trial. Eur J Anaesthesiol 2019; 36: 963-971. doi: 10.1097/EJA. 0000000000001110.

29. Kristensen MS, Abildstrøm HH. Endotracheal video-laryngoscope guided intubation with a $2.4 \mathrm{~mm}$ cuff'ed tube and active expiration by a dedicated ventilator versus a standard tube/ventilator. A ran domized single blinded study in patients with a predicted difficult airway. A paradigm shift in airway management? Euroanaesthesia 2019; 3755 [Abstract].

30. Meulemans J, Jans A, Vermeulen K, et al. Evone ${ }^{\circ}$ flow-controlled ventilation during upper airway surgery: a clinical feasibility study and safety assessment. Front Surg 2020; 7: 6. doi: 10.3389/fsurg. 2020.00006.

31. Weber J, Straka L, Borgmann S, Schmidt J, Writh S, Schumann S. Flow-controlled ventilation (FCV) improves regional ventilation in obese patients - a randomized controlled crossover trial. BMC Anesthesiol 2020; 20: 24. doi: 10.1186/s12871-020-0944-y.

32. Weber J, Schmidt J, Straka L, Wirth S, Schumann S. Flow-controlled ventilation improves gas exchange in lung-healthy patients - a randomized interventional cross-over study. Acta Anaesthesiol Scand 2020; 64: 481-488. doi: 10.1111/aas.13526.

33. Sebrechts T, Morrison SG, Schepens T, Saldien V. Flow-controlled ventilation with the Evone ventilator and Tritube versus volume-controlled ventilation: a clinical cross-over pilot study describing oxygenation, ventilation and haemodynamic variables. Eur J Anaesthesiol 2021; 38: 209-211. doi: 10.1097/EJA.0000000000001326.

34. Schmidt J, Wenzel C, Mahn M, et al. Improved lung recruitment and oxygenation during mandatory ventilation with a new expiratory ventilation assistance device: A controlled interventional trial in healthy pigs. Eur J Anaesthesiol 2018; 35: 736-744. doi: 10.1097/ EJA.0000000000000819.

35. Schmidt J, Wenzel C, Spassov S, et al. Flow-controlled ventilation attenuates lung injury in a porcine model of acute respiratory distress syndrome: a preclinical randomized controlled study. Crit Care Med 2020; 48: e241-e248. doi: 10.1097/CCM.0000000000004209.

36. Spraider, P, Martini J, Abram J, et al. Individualized flow-controlled ventilation compared to best clinical practice pressure-controlled ventilation: a prospective randomized porcine study. Crit Care 2020: 24: 662. doi: 10.1186/s13054-020-03325-3.

37. Barnes T, Enk D. Ventilation for low dissipated energy achieved using flow control during both inspiration and expiration. Trends in Anaesthesia and Critical Care 2019; 24: 5-12. doi: 10.1016/j.tacc. 2018.09.003

38. Nouraei SAR, Girgis M, Shorthouse J, El-Boghdadly K, Ahmad I. A multidisciplinary approach for managing the infraglottic difficult airway in the setting of the Coronavirus pandemic. Oper Tech Otolaryngol Head Neck Surg 2020; 31: 128-137. doi: 10.1016/j.otot. 2020.04.009.

39. Piazza C, Filauro M, Dikkers FG, et al. Long-term intubation and high rate of tracheostomy in COVID-19 patients might determine an unprecedented increase of airway stenoses: a call to action from the European Laryngological Society. Eur Arch Otorhinolaryngol 2020, 278: 1-7. doi: 10.1007/s00405-020-06112-6.

40. Magasich-Airola NP, Rosal Martins M, Desuter GR, Van Boven MJ Novel technique for safe tracheostomy during COVID-19 pandemi using Evone ${ }^{\infty}$ flow-controlled ventilation system. Int J Clin Pract 2021; 75: e13863. doi: 10.1111/ijcp.13863.

41. Shallik N, Elarref M, Khamash O, et al. Management of critical tracheal stenosis with a straw sized tube (Tritube): case report. Qatar Med J 2021: 2020: 48. doi: 10.5339/qmj.2020.48.

42. Wittenstein J, Scharffenberg M, Ran X, et al. Comparative effects of flow vs. volume-controlled one-lung ventilation on gas exchange and respiratory system mechanics in pigs. Intensive Care Med Exp 2020; 8 (Suppl 1): 24. doi: 10.1186/s40635-020-00308-0.

43. Neto AS, da Costa LGV, Hemmes SNT, et al. The LAS VEGAS risk score for prediction of postoperative pulmonary complications: An observational study. Eur J Anaesthesiol 2018; 35: 691-701. doi: 10.1097/EJA.0000000000000845.

44. Berlin DA, Manoach S, Oromendia C, Heerdt PM. Automated expiratory ventilation assistance through a small endotracheal tube can improve venous return and cardiac output. Intensive Care Med Exp 2019; 7: 6. doi: 10.1186/s40635-018-0217-y.

45. Böttinger L, van der Hoorn JWA. Negative pressure ventilation: a special application of expiratory ventilation assistance. Intensive Care Med Exp 2019; 7: 22. doi: 10.1186/s40635-019-0248-z.

46. Ordies S, Orlitova M, Heigl T, et al. Flow-controlled ventilation during EVLP improves oxygenation and preserves alveolar recruitment. Intensive Care Med Exp 2020; 8: 70. doi: 10.1186/s40635-020-00360-w.

47. Tsuboi N, Tsuboi K, Nosaka N, Nishimura N, Nakagawa S. The ventilatory strategy to minimize expiratory flow rate in ventilated patients with chronic obstructive pulmonary disease. Int J Chron Obstruct Pulmon Dis 2021; 16: 301-304. doi: 10.2147/COPD.S296343. 